\title{
Complete Quadratic Lyapunov functionals using Bessel-Legendre inequality
}

\author{
Alexandre Seuret $^{a, c}$ and Frédéric Gouaisbaut ${ }^{a, b}$
}

\begin{abstract}
The article is concerned with the stability analysis of time-delay systems using completeLyapunov functionals. This class of functionals has been employed in the literature because of their nice properties. Indeed, such a functional can be built if a system with a constant time delay is asymptotically stable. Hence, several articles aim at approximating their parameters thanks to a discretization method or polynomial modeling. The interest of such approximation is the design of tractable sufficient stability conditions expressed on the Linear Matrix Inequality or the Sum of Squares setups. In the present article, we provide an alternative method based on polynomial approximation which takes advantages of the Legendre polynomials and their properties. The resulting stability conditions are scalable with respect to the degree of the Legendre polynomials and are expressed in terms of a tractable LMI.
\end{abstract}

\section{INTRODUCTION}

Delays are inherent of real-time closed loop systems. Indeed, multiplication of networked controlled systems requires the explicit consideration of the communication delays since delays drastically affect the performances of the overall system. Hence, this practical problem motivates a large number of papers dedicated to the stability of linear time delay systems (see [6] and references therein). In the context of the stability analysis of such time-delay systems, the use of Lyapunov-Krasovskii functionals (LKF) has been very popular [4], [8], [11], [16], [18]. All theses papers focus on the choice of a particular structure for the LKF, which is usually composed by the sum of several typical terms [6], including quadratic function of the instantaneous state $x(t)$ and integral of quadratic functional of the entire delay state $x_{t}(\theta)$. In general, all theses functionals are particular case of the well-known complete Lyapunov-Krasovskii functionals (see Theorem 5.9 in [6]), given by

$$
\begin{aligned}
V\left(x_{t}\right)= & x^{T}(t) P x(t)+2 x^{T}(t) \int_{-h}^{0} Q(\theta) x_{t}(\theta) \mathrm{d} \theta \\
& +\int_{-h}^{0} \int_{-h}^{0} x_{t}\left(\theta_{1}\right) T\left(\theta_{1}, \theta_{2}\right) x_{t}\left(\theta_{2}\right) \mathrm{d} \theta_{1} \mathrm{~d} \theta_{2} \\
& +\int_{-h}^{0} x_{t}^{T}(\theta) S(\theta) x_{t}(\theta) \mathrm{d} \theta
\end{aligned}
$$

where $x_{t}(\theta)=x(t+\theta)$ represents the state of the time-delay system and $h>0$ the delay and where the

\footnotetext{
${ }^{a}$ CNRS, LAAS, 7 avenue du Colonel Roche, 31077 Toulouse, France. \{aseuret,fgouaisb\}@laas.fr

${ }^{b}$ Univ de Toulouse, UPS, LAAS, F-31400, Toulouse, France.

${ }^{c}$ Univ de Toulouse, LAAS, F-31400 Toulouse, France.

This work is supported in part by the ANR project LimICoS contract number 12 BS03 00501.
}

matrix $P$ is a symmetric positive definite and the matrix functions $Q, S$ and $T$ are differentiable (see Section 5.6.2 in [6] for more details). Theorem 5.9 from [6] ensures that, if the solutions of a time delay system is asymptotically stable, this functional is a LKF, provided that the different functions $Q$ and $T$ satisfy some partial differential equations, which is not an easy task especially for high dimensional delay systems. In practice, numerically checking the existence of such functionals often requires an approximation of this matrix functions in an appropriate manner. In [6] a discretization method was proposed where the functions $Q, T$ and $S$ where chosen piecewise linear and the conditions are presented through the LMI setup. In [12], these matrices where chosen as polynomials functions and the numerical test was performed using the SOSTOOLS.

In the present paper, we aim at presenting another approximation method which is also based on the polynomial approximation of the functions $Q$ and $T$ but using the particular setup of the Legendre polynomials. Indeed these polynomials are frequently used in the approximation theory because of their relevant properties which are described in the following section. Thanks to the introduction of these polynomials, we are able to provide a new integral inequality whose conservatism can be made arbitrarily small. This is the core tool for developing a set of new sufficient conditions indexed by $N$, the degree of the polynomials modeling the parameters of the complete LKF. It is proved also that this set forms an hierarchy with respect to the pair $(h, N)$ in the sense that increasing $N$ improves the result. Finally, two examples show the effectiveness of the method.

Notations: Throughout the paper $\mathbb{R}^{n}$ denotes the $n$-dimensional Euclidean space with vector norm $|\cdot|$, $\mathbb{R}^{n \times m}$ is the set of all $n \times m$ real matrices. The notation $P \succ 0$, for $P \in \mathbb{R}^{n \times n}$, means that $P$ is symmetric and positive definite. The set $\mathcal{S}_{n}^{+}$represents the set of symmetric positive definite matrices of $\mathbb{R}^{n \times n}$. The set of continuous functions from an interval $\mathcal{I} \subset \mathbb{R}$ to $\mathbb{R}^{n}$ which are square integrable is denoted as space $\mathcal{L}_{2}\left(\mathcal{I} \rightarrow \mathbb{R}^{n}\right)$. The symmetric matrix $\left[\begin{array}{cc}A & B \\ * & C\end{array}\right]$ stands for $\left[\begin{array}{cc}A & B \\ B^{T} & C\end{array}\right]$. $\operatorname{diag}(A, B)$ stands for the diagonal matrix $\left[\begin{array}{rr}A & 0 \\ 0 & B\end{array}\right]$. Moreover, for any square matrix $A \in \mathbb{R}^{n \times n}$, we define $\operatorname{He}(A)=A+A^{T}$. The matrix $I$ represents the identity matrix of appropriate dimension. The notation $0_{n, m}$ stands for the matrix in $\mathbb{R}^{n \times m}$ whose entries are 
zero and, when no confusion is possible, the subscript will be omitted. The notation $\left(\begin{array}{c}k \\ l\end{array}\right)$ refers to the binomial coefficients given by $\frac{k !}{(k-l) ! l !}$.

\section{NEW INTEGRAL INEQUALITIES}

\section{A. Legendre polynomials}

In this article, we aim at taking advantages of the Legendre polynomials to provide new integral inequalities and a new method to construct complete LyapunovKrasovskii functionals. Let first recall the definition and the basic properties of the Legendre polynomials.

Definition 1: The Legendre polynomials considered over the interval $[-h, 0]$ are defined by

$$
\forall k \in \mathbb{N}, \quad L_{k}(u)=(-1)^{k} \sum_{l=0}^{k} p_{l}^{k}\left(\frac{u+h}{h}\right)^{l},
$$

with $p_{l}^{k}=(-1)^{l}\left(\begin{array}{c}k \\ l\end{array}\right)\left(\begin{array}{c}k+l \\ l\end{array}\right)$.

Another reason for employing these polynomials comes from their nice properties that are summarized below, which will be useful in the latter developments.

Property 2: The Legendre polynomials described in Definition 1 satisfy the following properties:

P1 Orthogonality: $\forall(k, l) \in \mathbb{N}^{2}$,

$$
\int_{-h}^{0} L_{k}(u) L_{l}(u) \mathrm{d} u= \begin{cases}0, & k \neq l \\ \frac{h}{2 k+1}, & k=l\end{cases}
$$

P2 Boundary conditions:

$$
\forall k \in \mathbb{N}, \quad L_{k}(0)=1, \quad L_{k}(-h)=(-1)^{k} .
$$

P3 Differentiation: $\frac{\mathrm{d}}{\mathrm{d} u} L_{k}(u)=0$, if $k=0$, and

$$
\frac{\mathrm{d}}{\mathrm{d} u} L_{k}(u)=\sum_{i=0}^{k-1} \frac{(2 i+1)}{h}\left(1-(-1)^{k+i}\right) L_{i}(u),
$$

if $k \geq 1$.

Proofs of these properties can be found in [3].

\section{B. Bessel-Legendre inequalities}

Based on the Legendre polynomials and an application of Bessel's inequality [3], we obtain the following lemma.

Lemma 3: Let $x \in \mathcal{L}_{2}\left(\mathcal{I} \rightarrow \mathbb{R}^{n}\right)$ and $R \in \mathcal{S}_{n}^{+}$. The integral inequality

$$
\int_{-h}^{0} x(u) R x(u) \mathrm{d} u \geq \frac{1}{h}\left[\begin{array}{c}
\Omega_{0} \\
\vdots \\
\Omega_{N}
\end{array}\right]^{T} R_{N}\left[\begin{array}{c}
\Omega_{0} \\
\vdots \\
\Omega_{N}
\end{array}\right]
$$

holds, for all $N \in \mathbb{N}$, where

$$
\begin{aligned}
& R_{N}=\operatorname{diag}(R, 3 R, \ldots,(2 N+1) R) \\
& \Omega_{k}=\int_{-h}^{0} L_{k}(u) x(u) \mathrm{d} u, \text { for all } k \in \mathbb{N} .
\end{aligned}
$$

Proof: Consider a function $x$ in $\mathcal{L}_{2}\left(\mathcal{I} \rightarrow \mathbb{R}^{n}\right)$ and a matrix $R$ in $\mathcal{S}_{n}^{+}$. Define the function $z$ by

$$
z(u)=x(u)-\sum_{k=0}^{N} \frac{2 k+1}{h} \Omega_{k} L_{k}(u)
$$

From its definition, $z$ is in $\mathcal{L}_{2}\left(\mathcal{I} \rightarrow \mathbb{R}^{n}\right)$ and the integral $\int_{-h}^{0} z^{T}(u) R z(u) d u$ exists. From the orthogonal property of the Legendre polynomials, one has

$$
\begin{aligned}
\int_{-h}^{0} z^{T}(u) & R z(u) d u=\int_{-h}^{0} x^{T}(u) R x(u) d u \\
& -2 \sum_{k=0}^{N} \frac{2 k+1}{h}\left(\int_{-h}^{0} L_{k}(u) x(u) d u\right) R \Omega_{k} \\
& +\sum_{k=0}^{N}\left(\frac{2 k+1}{h}\right)^{2} \int_{-h}^{0} L_{k}^{2}(u) d u \Omega_{k}^{T} R \Omega_{k}
\end{aligned}
$$

Finally by noting that $\Omega_{k}=\int_{-h}^{0} L_{k}(u) x(u) \mathrm{d} u$ and $\left(\frac{2 k+1}{h}\right)^{2} \int_{-h}^{0} L_{k}^{2}(u) d u=\frac{2 k+1}{h}$, it yields

$$
\begin{gathered}
\int_{-h}^{0} z^{T}(u) R z(u) d u=\int_{-h}^{0} x^{T}(u) R x(u) d u \\
-\sum_{k=0}^{N} \frac{2 k+1}{h} \Omega_{k}^{T} R \Omega_{k},
\end{gathered}
$$

The proof is concluded by noting that, since $R \succ 0$, the left hand side of the previous equation is positive definite, which directly implies the inequality (3).

In the remainder of this paper, inequality (3) will be recalled as Bessel-Legendre (B-L) Inequality.

Remark 1: The previous inequality encompasses the Jensen inequality [6] and the recent Wirtinger-based integral inequality [15] as the particular cases $N=0$ and $N=1$, respectively. Thus, the set of inequalities provided in Lemma 3 represents more general formulation than these two inequalities. Additionally, the Parseval identity proves that the inequalities (3) becomes non conservative as $N$ goes to $\infty$.

\section{ApPlicAtion to THE STABility ANALYSis OF SYSTEMS WITH A DISCRETE DELAY}

Consider a linear time-delay system of the form:

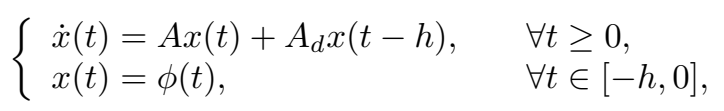

where $x(t) \in \mathbb{R}^{n}$ is the state vector, $\phi$ is a continuous functions, which represents the initial conditions and $A$ and $A_{d}$, are constant matrices. The delay is assumed to be constant. In this section, we will show how the previous inequalities can be applied to the stability analysis of time delay systems.

\section{A. Choice of a Lyapunov-Krasovskii functional}

In this subsection, we aim at choosing a new structure for the LKF based on the use of Legendre polynomials. Considering the LKF of the form (1), we propose to model the different matrices $P, Q(\theta), T(\theta, s)$ and $S(\theta)$ as polynomials with respect to the variables $\theta$ and $s$. Contrary to an SOS formulation [12], these polynomials are expressed in terms of the Legendre basis as follows:

$$
Q(\theta)=\sum_{i=0}^{N} Q_{i} L_{i}(\theta), T(\theta, s)=\sum_{i=0}^{N} \sum_{j=0}^{N} L_{i}(\theta) L_{j}(s) T_{i j}
$$

where the matrices $Q_{i}, T_{i j}=T_{j i}^{T}, i \in\{0, \ldots, N\}, j \in$ $\{0, \ldots, N\}$ have to be optimized. The polynomial matrix $S(\theta)$ is chosen as a linear function with respect to $\theta$ and is therefore simply expressed with the canonical basis.

$$
S(\theta)=S+(h+\theta) R .
$$


Hence, the functional $V_{N}$ is rewritten as :

$$
\begin{aligned}
& V_{N}\left(x_{t}\right)=\tilde{x}_{N}^{T}(t) P_{N} \tilde{x}_{N}(t) \\
& \quad+\int_{t-h}^{t} x^{T}(s)(S+(h-t+s) R) x(s) \mathrm{d} s,
\end{aligned}
$$

where

$$
P_{N}=\left[\begin{array}{cccc}
P & Q_{0} & \ldots & Q_{N} \\
Q_{0}^{T} & T_{00} & \ldots & T_{0 N} \\
\vdots & \vdots & & \vdots \\
Q_{N}^{T} & T_{N 0} & \ldots & T_{N N}
\end{array}\right],
$$

and where extended state defined as:

$$
\tilde{x}_{N}(t)=\left[\begin{array}{c}
x_{t}(0) \\
\int_{-h}^{0} L_{0}(s) x_{t}(s) \mathrm{d} s \\
\vdots \\
\int_{-h}^{0} L_{N}(s) x_{t}(s) \mathrm{d} s
\end{array}\right], \quad N \geq 0,
$$

collects the current state and the projections of the state function $x_{t}$ to the $N$ first Legendre polynomials.

\section{B. Systems with constant and known delay}

We present in this sub-section a first stability result for time-delay systems, which is based on the proposed LKF (5) and the use of the B-L inequality developed in the previous section. Based on the previous inequalities, the following stability theorem is provided by the use of Lemma 3 with an arbitrary $N$.

Theorem 4: For a given integer $N \geq 1$ and a constant delay $h$, assume that there exist a matrix $P_{N} \in \mathcal{S}_{(N+1) n}^{+}$, and two matrices $S, R \in \mathcal{S}_{n}^{+}$such that the following LMI is satisfied

$$
\Phi_{N}^{+}(h):=P_{N}+\frac{1}{h} \operatorname{diag}(0, S, 3 S, \ldots,(2 N-1) S) \succ 0,
$$

and

$$
\Phi_{N}^{-}(h)=\Phi_{N 0}(h)-\frac{1}{h} \operatorname{diag}\left(0,0, R_{N}\right) \prec 0,
$$

where

$$
\begin{aligned}
& \Phi_{N 0}(h)=\operatorname{He}\left(G_{N}^{T}(h) P H_{N}\right)+\tilde{S}_{N}(h), \\
& \tilde{S}_{N}(h)=\operatorname{diag}\left\{S+h R,-S, 0_{(N+1) n}\right\}, \\
& G_{N}(h)=\left[\begin{array}{ccc}
I & 0_{n} & 0_{n, n(N+1)} \\
0_{n(N+1), n} & 0_{n(N+1), n} & h I_{n(N+1)}
\end{array}\right], \\
& H_{N}=\left[\begin{array}{lllll}
F_{N}^{T} & \Gamma_{N}^{T}(0) & \Gamma_{N}^{T}(1) & \ldots & \Gamma_{N}^{T}(N)
\end{array}\right]^{T},
\end{aligned}
$$

and where

$$
\begin{aligned}
& F_{N}=\left[\begin{array}{lll}
A & A_{d} & 0_{n, n(N+1)},
\end{array}\right], \\
& \Gamma_{N}(k)=\left[\begin{array}{lllll}
I & (-1)^{k+1} I & \gamma_{N k}^{0} I & \ldots & \gamma_{N k}^{N} I
\end{array}\right], \\
& \gamma_{N k}^{i}= \begin{cases}-(2 i+1)\left(1-(-1)^{k+i}\right), & \text { if } i \leq k, \\
0, & \text { if } i \geq k+1 .\end{cases}
\end{aligned}
$$

Then the time delay system (4) is asymptotically stable for the constant delay $h$.

Proof: Consider the LKF (5), since $S \succ 0$ and following the procedure provided in [6], Lemma 3 can be applied to the second term of $V_{N}$ to give a lower bound of the functional. In order to be consistent with the definition of $\tilde{x}_{N}$, Lemma 3 is considered with the order $N$. It thus yields

$$
\begin{aligned}
V_{N}\left(x_{t}\right) \geq & \tilde{x}_{N}^{T}(t) \Phi_{N}^{+}(h) \tilde{x}_{N}(t) \\
& +\int_{t-h}^{t}(h-t+s) x^{T}(s) R x(s) \mathrm{d} s .
\end{aligned}
$$

Then, the positive definiteness of $V_{N}$ results from the condition $S \succ 0, R \succ 0$ and $\Phi_{N}^{+} \succ 0$.

Let us concentrate on the differentiation of the functionals along the trajectories of the system. To do so, let us first define the vector

$$
\xi_{N}^{T}(t)=\left[\begin{array}{c}
x_{t}(0) \\
x_{t}(-h) \\
\frac{1}{h} \int_{-h}^{0} L_{0}(s) x_{t}(s) \mathrm{d} s \\
\vdots \\
\frac{1}{h} \int_{-h}^{0} L_{N}(s) x_{t}(s) \mathrm{d} s
\end{array}\right], \quad N \geq 0,
$$

which will be employed to expressed the derivative of the functional. The computation of $\dot{V}_{N}$ leads to

$$
\begin{aligned}
\dot{V}_{N}\left(x_{t}\right) & =2 \tilde{x}_{N}^{T}(t) P_{N} \dot{\tilde{x}}_{N}(t)+x_{t}^{T}(0)(S+h R) x_{t}(0) \\
& -x_{t}^{T}(-h) S x_{t}(-h)-\int_{-h}^{0} x_{t}^{T}(s) R x_{t}(s) \mathrm{d} s,
\end{aligned}
$$

where

$$
\dot{\tilde{x}}_{N}^{T}(t)=\left[\begin{array}{c}
\dot{x}_{t}(0) \\
\int_{-h}^{0} L_{0}(s) \dot{x}_{t}(s) \mathrm{d} s \\
\vdots \\
\int_{-h}^{0} L_{N}(s) \dot{x}_{t}(s) \mathrm{d} s
\end{array}\right] .
$$

The following setup consists of the expression of the vector $\dot{\tilde{x}}_{N}(t)$ using the augmented vector $\xi_{N}(t)$. On the first hand, it is clear that

$$
\dot{x}_{t}(0)=A_{0} x_{t}(0)+A_{1} x_{t}(-h)=F_{N} \xi_{N}(t) .
$$

On the other hand, for any positive integer $k \leq N$, an integration by parts ensures that

$$
\begin{aligned}
\int_{-h}^{0} L_{k}(s) \dot{x}_{t}(s) \mathrm{d} s= & L_{k}(0) x_{t}(0)-L_{k}(-h) x_{t}(-h) \\
& -\int_{-h}^{0} \dot{L}_{k}(u) x_{t}(u) \mathrm{d} u .
\end{aligned}
$$

Thanks to properties P2 and P3 of the Legendre polynomials, the following expression is derived

$$
\begin{aligned}
\int_{-h}^{0} L_{k}(s) \dot{x}_{t}(s) \mathrm{d} s= & x_{t}(0)-(-1)^{k} x_{t}(-h) \\
& -\sum_{i=0}^{k-1} \gamma_{N k}^{i} \int_{-h}^{0} L_{i}(u) x(u) \mathrm{d} u \\
= & \Gamma_{N}(k) \xi_{N}(t) .
\end{aligned}
$$

Then, by putting together all the components of $\dot{\tilde{x}}_{N}(t)$, we obtain

$$
\dot{\tilde{x}}_{N}(t)=H_{N}(h) \xi_{N}(t)
$$

Finally, by noting that $\tilde{x}_{N}(t)=G_{N}(h) \xi_{N}(t)$, it yields

$$
\dot{V}_{N}\left(x_{t}, \dot{x}_{t}\right)=\xi_{N}^{T}(t) \Phi_{N 0}(h) \xi_{N}(t)-\int_{-h}^{0} x_{t}^{T}(s) R x_{t}(s) \mathrm{d} s .
$$

Applying Lemma 3 to the order $N$ ensures that

$$
-\int_{-h}^{0} x_{t}^{T}(s) R x_{t}(s) \mathrm{d} s \leq-\frac{1}{h} \xi_{N}^{T}(t) \operatorname{diag}\left(0,0, R_{N}\right) \xi_{N}(t) .
$$

Reinjecting this inequality into (9) leads to $\dot{V}_{N}\left(x_{t}, \dot{x}_{t}\right) \leq$ $\xi_{N}^{T}(t) \Phi_{N}^{-}(h) \xi_{N}(t)$. Hence, if the LMI (7) is satisfied, the delay system (4) is asymptotically stable for the constant delay $h$.

Remark 2: It is interesting to notice that starting from a particular choice for a complete LKF, the proposed 
stability criterion is equivalent to consider the stability of the following system:

$$
\begin{cases}\dot{x}(t) & =A_{0} x(t)+A_{1} x(t-h) \\ \int_{-h}^{0} L_{0}(s) \dot{x}_{t}(s) \mathrm{d} s & =x(t)-x(t-h) \\ \vdots & \\ \int_{-h}^{0} L_{N}(s) \dot{x}_{t}(s) \mathrm{d} s & =x(t)-(-1)^{k} x(t-h) \\ & -\sum_{i=0}^{k-1} \gamma_{N k}^{i} \int_{-h}^{0} L_{i}(u) x(u) \mathrm{d} u .\end{cases}
$$

studied with the basic LKF.

$$
\begin{aligned}
V_{N}\left(x_{t}\right) & =\tilde{x}_{N}^{T}(t) P_{N} \tilde{x}_{N}(t) \\
& +\int_{t-h}^{t} x^{T}(s)(S+(h-t+s) R x(s) \mathrm{d} s .
\end{aligned}
$$

Hence, the main tool which allows to deal with such a simplified LKF is the Bessel inequality, which connects the different states together. Indeed, this inequality links the $L_{2}[-h, 0]$ norm of the original delay state $x_{t}(\theta)$ with the $L_{2}[-h, 0]$ norms of its projection onto the set of polynomial of degree less than $N$.

\section{Delay range stability}

In Theorems 4 , the delay is supposed to be perfectly known. Therefore, it ensures stability of the delay system only for the pointwise delay $h$. The following subsection extends this first result by considering that the delay $h$ is unknown but belongs to a prescribed interval $\left[h_{1}, h_{2}\right]$. We aim therefore at providing a criterion which ensures stability for all constant delays in this pocket.

Theorem 5: For a given integer $N$ and an uncertain constant delay $h \in\left[h_{1}, h_{2}\right]$, assume that there exist a matrix $P_{N}=P_{N}^{T} \in \mathcal{R}_{(N+1) n \times(N+1) n}$ and two matrices $S, R \in \mathcal{S}_{n}^{+}$such that the LMIs $\Phi_{N}^{+}\left(h_{2}\right) \succ 0$ and

$$
\Psi_{N}\left(h, h_{2}\right)=\Psi_{N 0}\left(h, h_{2}\right)-\frac{1}{h_{2}} \operatorname{diag}\left(0,0, R_{N}\right) \prec 0
$$

hold for $h=\left\{h_{1}, h_{2}\right\}$, where

$$
\Psi_{N 0}\left(h, h_{2}\right)=\operatorname{He}\left(G_{N}^{T}(h) P H_{N}\right)+\tilde{S}_{N}\left(h_{2}\right),
$$

and where $\tilde{S}_{N}, F_{N}, G_{N}, H_{N}$ and $\Gamma_{N}(k)$ are defined in Theorem 4 . Then the time delay system (4) is asymptotically stable for any constant delay $h$ in the interval $\left[h_{1}, h_{2}\right]$.

\section{Proof: Consider the LKF given by}

$$
\begin{aligned}
\tilde{V}_{N}\left(x_{t}, \dot{x}_{t}\right)= & \tilde{x}_{N}^{T}(t) P_{N} \tilde{x}_{N}(t)+\int_{t-h}^{t} x^{T}(s) S x(s) \mathrm{d} s \\
& +\int_{t-h_{2}}^{t}\left(h_{2}-t+s\right) x^{T}(s) R x(s) \mathrm{d} s
\end{aligned}
$$

where $\tilde{x}_{N}(t)$ has the same definition as in the proof of Theorem 4 . The only difference with respect to the constant and known delay appears in the definition of the last term of $V_{N}$ which is defined with the delay $h_{2}$ instead of $h$. Following the proof of Theorem 4, it yields

$$
\begin{aligned}
\dot{\tilde{V}}_{N}\left(x_{t}, \dot{x}_{t}\right)= & \xi_{N}^{T}(t) \Psi_{N 0}\left(h, h_{2}\right) \xi_{N}(t) \\
& -\int_{-h_{2}}^{0} x^{T}(t+s) R x(t+s) \mathrm{d} s \\
\leq & \xi_{N}^{T}(t) \Psi_{N 0}\left(h, h_{2}\right) \xi_{N}(t) \\
& -\int_{-h}^{0} x^{T}(t+s) R x(t+s) \mathrm{d} s .
\end{aligned}
$$

Applying Lemma 3 to the order $N$ leads to

$$
\dot{\tilde{V}}_{N}\left(x_{t}, \dot{x}_{t}\right) \leq \xi_{N}^{T}(t) \Psi_{N}\left(h, h_{2}\right) \xi_{N}(t) .
$$

By noting that $\Psi_{N}\left(h, h_{2}\right)$ is affine in $h$, it is easy to see that

$$
\Psi_{N}\left(h, h_{2}\right)=\frac{h-h_{1}}{h_{2}-h_{1}} \Psi_{N}\left(h_{2}, h_{2}\right)+\frac{h_{2}-h}{h_{2}-h_{1}} \Psi_{N}\left(h_{1}, h_{2}\right) .
$$

Hence, it suffices to ensure $\Psi_{N}\left(h_{1}, h_{2}\right) \prec 0$ and $\Psi_{N}\left(h_{2}, h_{2}\right) \prec 0$ to guarantee that the system is asymptotically stable for all constant delay $h \in\left[h_{1} h_{2}\right]$.

\section{Hierarchy of LMI Stability CONDitions}

This section aims at proving that the previous stability conditions form a hierarchy of LMI conditions. This is formulated in the following theorem based on the stability conditions of Theorem 4 .

Theorem 6: For any time delay system (4), define the set $\mathcal{H}_{N}$ by

$$
\begin{aligned}
\mathcal{H}_{N}:= & \left\{h>0: \exists\left(P_{N}, S(N), R(N)\right) \in \mathcal{S}_{(N+1) n}\right. \\
& \left.\times\left(\mathcal{S}_{n}^{+}\right)^{2}, \quad \text { s.t. } \quad \Phi_{N}^{+}(h) \succ 0, \Phi_{N}^{-}(h) \prec 0\right\} .
\end{aligned}
$$

Then, $\mathcal{H}_{N} \subset \mathcal{H}_{N+1}$ holds, for all $N \geq 0$.

Proof: Let $N \in \mathbb{N}$. If $\mathcal{H}_{N}$ is empty, the inclusion is trivial. Assume that $\mathcal{H}_{N}$ is not empty and consider an element $h \in \mathcal{H}_{N}$. From the definition of $\mathcal{H}_{N}$, there exist $P_{N}=P_{N}^{T}, S(N) \succ 0$ and $R(N) \succ 0$ such that $\Phi_{N}^{+}(h) \succ 0$ and $\Phi_{N}^{-}(h) \prec 0$. Taking advantages of the construction of the LKF (5), we suggest the matrices

$$
\begin{gathered}
P_{N+1}=\left[\begin{array}{cc}
P_{N} & 0 \\
0 & \epsilon I
\end{array}\right], \\
S(N+1)=S(N)=S, \\
R(N+1)=R(N)=R,
\end{gathered}
$$

where $\epsilon>0$ is a scalar to be chosen. Clearly this choice of matrices ensures that the functional $V_{N+1}$ is positive definite. According to the construction of the matrices $G_{N}, H_{N}, F_{N}$ and $\tilde{S}_{N}$, the following relation holds

$$
\begin{aligned}
H_{N+1} & =\left[\begin{array}{cc}
H_{N} & 0_{N n, n} \\
\Gamma_{N+1}(N+1)
\end{array}\right], \\
G_{N+1}(h) & =\left[\begin{array}{cc}
G_{N}(h) & 0_{N n, n} \\
0_{n, N n} & h I
\end{array}\right], \\
\tilde{S}_{N+1} & =\left[\begin{array}{cc}
\tilde{S}_{N} & 0_{N n, n} \\
0_{n, N n} & 0_{n}
\end{array}\right], \\
F_{N+1} & =\left[\begin{array}{ll}
F_{N} & 0_{n}
\end{array}\right],
\end{aligned}
$$

From these expressions, the matrix $\Phi_{N+1}(h)$ can be expressed using the matrix $\Phi_{N}^{+}(h)$ as follows

$$
\begin{aligned}
\Phi_{N+1}^{+}(h)= & {\left[\begin{array}{cc}
\Phi_{N}(h) & 0 \\
0 & -(2 N+3) R
\end{array}\right] } \\
& +\epsilon H e\left\{h \Gamma_{N+1}^{T}(N+1)\left[\begin{array}{l}
0 \\
I
\end{array}\right]\right\} .
\end{aligned}
$$

Then, since $\Phi_{N}^{+}(h) \prec 0, R \succ 0$, the first term of the previous expression is negative definite. It implies that there exists a sufficiently small $\epsilon$ for which $\Phi_{N+1}^{+}(h) \prec 0$, 
which proves that $h$ belongs to $\mathcal{H}_{N+1}$, which allows to conclude that $\mathcal{H}_{N} \subset \mathcal{H}_{N+1}$.

Since Theorem 4 only provides sufficient stability condition, the sequence of sets $\left\{\mathcal{H}_{N}\right\}_{N \in \mathbb{N}}$ is an increasing sequence of set representing an inner approximation of the stability pockets. However, the previous theorem does not prove that the conditions of Theorem 4 will converge to the analytical bounds of the delay.

An analogous theorem showing that Theorem 5 also forms a hierarchy of stability conditions can be obtained.

\section{EXAMPLES}

The purpose of the following section is to illustrate our propositions on an academic and also a non trivial example. Because of space limitations, only the results provided by Theorem 4 are presented.

\section{A. Example 1}

First, consider the well known academic example of the form (4) with the matrices

$$
A=\left[\begin{array}{cc}
-2 & 0 \\
0 & -0.9
\end{array}\right], \quad A_{d}=\left[\begin{array}{cc}
-1 & 0 \\
-1 & -1
\end{array}\right] .
$$

It can be proved by direct inspection of the characteristic equation that this delay system is stable for all delays belonging to $[0,6.1725]$. To illustrate the main theorem, Table I compares the upperbound calculated by Theorem 4 with those found in the literature. All papers except [9] use Lyapunov theory in order to derive stability criteria. Some results based on Jensen lemma bounding technique gives nearly the same results [4], [8], [16], [18]. Other methods, which employ an augmented Lyapunov functional (with triple integral term) can go further but with a numerically increasing burden. The partitioning approach proposed by [7] based on the discrete delay decomposition of a simple LKF is very efficient and goes along with an important numerical complexity. Two techniques [5],[12] are based on the structure of the complete quadratic functional. Using an SOS optimisation setup, [12] approximates the matrices (which constitute the complete LKF (1)) as polynomials of a prescribed upperbound. [5] proposes a partionning complete Quadratic LKF along with a linear modeling of the matrices. As expected, all the two methods give very good results results with a similar numerical burden.

1) Example 2: This example is taken from the dynamics modeling of machining chatter [20], [17] and has been barely studied in the literature of time delay system using the Lyapunov-Krasovskii Theorem and LMI conditions. We consider:

$$
\left\{\begin{array}{l}
\dot{x}(t)=A x(t)+B u(t) \\
y(t)=C x(t)
\end{array}\right.
$$

with

$$
A=\left[\begin{array}{cccc}
0 & 0 & 1 & 0 \\
0 & 0 & 0 & 1 \\
-10 & 10 & 0 & 0 \\
5 & -15 & 0 & -0.25
\end{array}\right], \quad B=\left[\begin{array}{l}
0 \\
0 \\
1 \\
0
\end{array}\right], C=\left[\begin{array}{l}
1 \\
0 \\
0 \\
0
\end{array}\right]^{T}
$$

A delayed static output feedback controller is proposed:

$$
u(t)=-K y(t)+K y(t-h)
$$

where $K$ is the gain of the controller and $h$ is an unknown constant delay. The resulting dynamics is thus modeled by a time-delay system:

$$
\dot{x}(t)=A_{0} x(t)+A_{1} x(t-h),
$$

with $A_{0}=A-B C K$ and $A_{1}=B C K$. Considering $K=1$ and $h=3$, we are interesting firstly whether the classical methods of the literature could prove the stability and secondly, if so, to determine their computational complexity. Surprisingly, the results based on Jensen lemma do not prove the stability as, also, the partitioning method of [7]. The discretized Complete LKF of [5] is not able to guarantee stability with a discretization step lower than $N=12$ (we did not look for higher levels, because of the computation time). The SOS optimization of [12] requires a relaxation of order $N=10$, which corresponds to consider for the matrices $R, Q, S$ as polynomials of order 10 . Our proposal gives a positive answer only for $N=5$.

\section{Discussions}

Inspecting Example 1, the stability conditions provided in Theorem 4 do not seem very competitive, in terms of complexity, with respect to the most efficient stability conditions (Discretization and Sum of Squares methods). However, this method is very effective on the second example when assessing stability is not trivial. Additionally, the method based on Legendre polynomials has a relevant feature. Indeed, Theorem 6 theoretically and elegantly proves that increasing the degree $N$ of the polynomials always helps in reducing the conservatism. To the best of our knowledge, this type of result has not been addressed frequently in the literature.

In order to improve the efficiency of our method, the following problems have to be addressed in future works.

a) A method to reduce the conservatism to be more competitive with existing stability conditions must be provided. One direction would be to slightly modify the Lyapunov-Krasovskii functional. Indeed, it is well-known that the following integral quadratic term

$$
V_{0}\left(\dot{x}_{t}\right)=\int_{t-h}^{t}(h-t+s) \dot{x}^{T}(s) R \dot{x}(s) \mathrm{d} s,
$$

significantly helps to reduce the conservatism. This is the core of the work presented in [14], [15], which indeed shows an impressive reduction of the conservative with respect to the stability conditions of this article.

b) Another key issue is also to extend the proposed results to delay which are not constant. Indeed it is not clear, at least for the authors whether our method based on the Legendre polynomials could be easily adapted to the situation when the delay is time-varying. 


\begin{tabular}{|c|c|c||c|c|c|}
\hline Theorems & $h_{\max }$ & number of variables & Theorems & $h_{\max }$ & number of variables \\
\hline \hline$[4],[8],[16],[18]$ & 4.472 & $1.5 n^{2}+1.5 n$ & {$[5], D_{d}=1$} & 6.053 & $7.5 n^{2}+3.5 n$ \\
{$[9]$} & 6.1107 & $1.5 n^{2}+9 n+9$ & {$[5], D_{d}=2$} & 6.165 & $10.5 n^{2}+4.5 n$ \\
{$[2]$} & 5.120 & $7 n^{2}+4 n$ & {$[5], D_{d}=3$} & 6.171 & $14.5 n^{2}+4.5 n$ \\
{$[18]$} & 5.02 & $18 n^{2}+18 n$ & {$[5], D_{d}=4$} & 6.171 & $20.5 n^{2}+5.5 n$ \\
{$[10]$} & 4.97 & $69 n^{2}+5 n$ & {$[12], D_{p}=1$} & 5.19 & $7 n^{2}+3 n$ \\
{$[1]$} & 5.120 & $6.5 n^{2}+3.5 n$ & {$[12], D_{p}=2$} & 5.90 & $12.5 n^{2}+4.5 n$ \\
{$[19]$} & 5.30 & $8.5 n^{2}+3.5 n$ & {$[12], D_{p}=3$} & 6.10 & $21 n^{2}+6 n$ \\
{$[7], D_{d}=2$} & 5.71 & $4 n^{2}+2 n$ & Th.4,$N=2$ & 3.21 & $9 n^{2}+3 n$ \\
{$[7], D_{d}=3$} & 5.96 & $6.5 n^{2}+2.5 n$ & Th.4, $N=4$ & 5.28 & $19 n^{2}+4 n$ \\
{$[7], D_{d}=4$} & 6.05 & $10 n^{2}+3 n$ & Th.4, $N=6$ & 6.12 & $33 n^{2}+5 n$ \\
{$[13]$} & 5.901 & $3 n^{2}+2 n$ & Th. $4, N=8$ & 6.1725 & $51 n^{2}+6 n$ \\
\hline
\end{tabular}

TABLE I: Results for Example (14) for constant delay $h$. The notations $D_{d}$ and $D_{p}$ stands for the the degree of discretization and for the degree of the polynomial, respectively.

c) The set of polynomials is dense in the set of continuous functions defined on compact set, which implies

$$
\int_{-h}^{0} x^{T}(u) R x(u) d u=\sum_{k=0}^{\infty} \frac{2 k+1}{h} \Omega_{k}^{T}(u) R \Omega_{k} .
$$

Then, since the only conservatism in the proof of Theorem 4 appears when employing the integral inequality provided in Lemma 3, there is some hope to prove that the conservatism can be arbitrarily reduced. This would mean that our proposal could lead to an asymptotically necessary and sufficient stability condition. Of course, this is still a result to be proven.

\section{ViI. Conclusions}

In this paper, we provide a novel method to construct complete Quadratic Lyapunov-Krasovskii functionals proposed originally by [5]. The matrices, which constitute the functional, are chosen to be polynomials and are expressed with the help of the Legendre polynomials basis. This new functional can also be viewed as a new simple Lyapunov-Krasovskii functional for an extended state composed by the instantaneous state $x_{t}(0)$ and the projection of the state $x_{t}(\theta)$ onto the polynomial Legendre set with respect to a well defined inner product. An extensive use of Bessel inequality allows to develop efficient criteria, at least on examples, but with a large numerical complexity. This set of stability conditions forms a hierarchy of LMI indexed by the polynomial degree $N$, in the sense that increasing $N$ reduces the conservatism of the proposed method. Future works will include the study of the asymptotic necessity of this approach.

\section{REFERENCES}

[1] Y. Ariba and F. Gouaisbaut. An augmented model for robust stability analysis of time-varying delay systems. Int. J. Control, 82:1616-1626, 2009.

[2] Y. Ariba, F. Gouaisbaut, and K.H. Johansson. Stability interval for time-varying delay systems. In Decision and Control (CDC), 2010 49th IEEE Conference on, pages 1017 $-1022,2010$.

[3] W. Gautschi. Orthogonal Polynomials, Computation and Approximation. Oxford Science, 2004.

[4] F. Gouaisbaut and D. Peaucelle. A note on stability of time delay systems. In $5^{\text {th }}$ IFAC Symposium on Robust Control Design (ROCOND'06), Toulouse, France, 2006.
[5] K. Gu. A further refinement of discretized Lyapunov functional method for the stability of time-delay systems. Int. Journal of Control, 74(10):967 - 976, 2001.

[6] K. Gu, V. L. Kharitonov, and J. Chen. Stability of Time-Delay Systems. Birkhäuser Boston, 2003. Control engineering.

[7] Q.-L. Han. A discrete delay decomposition approach to stability of linear retarded and neutral systems. Automatica, 45(2):517 - 524, 2009.

[8] Y. He, Q. G. Wang, L. Xie, and C. Lin. Further improvement of free-weighting matrices technique for systems with timevarying delay. IEEE Trans. on Automat. Control, 52(2):293299, 2007.

[9] C.Y. Kao and A. Rantzer. Stability analysis of systems with uncertain time-varying delays. Automatica, 43(6):959 - 970, 2007.

[10] J.H. Kim. Note on stability of linear systems with time-varying delay. Automatica, 47(9):2118-2121, 2011.

[11] S.-I. Niculescu. Delay Effects on Stability. A Robust Control Approach. Springer-Verlag, 2001.

[12] M.M. Peet, A. Papachristodoulou, and S. Lall. Positive forms and stability of linear time-delay systems. SIAM Journal on Control and Optimization, 47(6):3227-3258, 2009.

[13] A. Seuret and F. Gouaisbaut. On the use of the Wirtinger's inequalities for time-delay systems. In $10^{\text {th }}$ IFAC Workshop on Time Delay Systems (IFAC TDS'12), Boston, MA, USA, 2012.

[14] A. Seuret and F. Gouaisbaut. Hierarchy of LMI-conditions for the stability of time delay systems. Submitted to Automatica, 2014.

[15] A. Seuret and F. Gouaisbaut. Wirtinger-based integral inequality: Application to time-delay systems. Automatica, 49(9):2860-2866, 2013.

[16] H. Shao. New delay-dependent stability criteria for systems with interval delay. Automatica, 45(3):744 - 749, 2009.

[17] R. Sipahi, S. Niculescu, C.T. Abdallah, W. Michiels, and K. Gu. Stability and stabilization of systems with time delay. Control Systems, IEEE, 31(1):38 -65, 2011.

[18] J. Sun, G.P. Liu, J. Chen, and D. Rees. Improved delayrange-dependent stability criteria for linear systems with timevarying delays. Automatica, 46(2):466 - 470, 2010.

[19] J. Sun, G.P. Liu, and J. Chen. Delay-dependent stability and stabilization of neutral time-delay systems. International Journal of Robust and Nonlinear Control, 19(12):1364-1375, 2009.

[20] J. Zhang, C. R. Knopse, and P. Tsiotras. Stability of timedelay systems: Equivalence between Lyapunov and scaled small-gain conditions. IEEE Trans. on Automat. Control, 46(3):482-486, 2001. 